\title{
3 \\ DISPOSICIONES ESENCIALES DE LOS PROFESORES EN LAS RELACIONES CON SUS ALUMNOS DESDE UNA PERSPECTIVA ÉTICA-PEDAGÓGICA
}

\author{
(ESSENTIAL DISPOSITIONS OF TEACHERS IN THE RELATIONS WITH \\ THEIR STUDENTS FROM AN ETHICAL-PEDAGOGICAL PERSPECTIVE)
}

José Antonio Jordán Sierra

Universidad Autónoma de Barcelona

\section{RESUMEN}

Este artículo analiza, de forma amplia y propedéutica, algunas actitudes ético-pedagógicas profundas que muestran los profesores más auténticos cuando en la realidad práctica diaria se relacionan con sus alumnos. Para este estudio se han elegido tres disposiciones esenciales, siguiendo la propuesta de Van Manen: el amor, la responsabilidad y la esperanza pedagógicas. Las reflexiones del autor en este artículo se basan, especialmente, en propuestas de Van Manen y en investigaciones en las que el autor ha estado implicado últimamente sobre estos temas, utilizando una metodología fenomenológica-hermenéutica. Entre las aportaciones de este artículo, cabe destacar tres: en primer lugar, la incalculable influencia que esas actitudes densas de los profesores ejercen en la mejora académica y formativa de sus alumnos; en segundo lugar, la constatación de que sólo los profesores que poseen en grado notorio ese tipo de actitudes profundas gozan de una plena satisfacción en el ejercicio de su docencia; y, finalmente, la urgente necesidad de acentuar la formación inicial y permanente de los profesores en actitudes densas éticas-pedagógicas.

\section{ABSTRACT}

This article analyzes, from a wide and introductory approach, some deep attitudes from the ethical-pedagogical perspective which real and very authentic teachers show when they relate to their pupils in the practical daily reality. Three 
essential dispositions have been chosen for this study, following Van Manen's proposal: pedagogical love, responsibility and hope. The author's reflections in this article are based specially on Van Manen's proposals and in some researches in which he has been involved lately in relation with this theme, using the phenomenological-hermeneutical methodology. Some basic lines of one of them are mentioned, within this paper, with an illustrative purpose. Among the contributions of this article it is necessary to distinguish three: firstly, the incalculable influence that teachers deep attitudes exert in the academic and formative improvement of their pupils; secondly, the ascertainment that only the teachers who possess in high degree this type of deep attitudes enjoy a full satisfaction in the exercise of its teaching; finally, the urgent necessity to underline the initial and inservice training of the teachers in deep ethical-pedagogical attitudes.

\section{INTRODUCCIÓN}

El primer comentario a realizar se centra en el sentido y alcance que tiene el estudio que aquí comienza. Su objetivo, en efecto, no consiste en dar a conocer de forma intensa, específica o monográfica alguna investigación concreta que verse sobre los tres temas que se abordan en él, o sobre alguno de ellos de modo particular. El cometido primordial del presente artículo no es otro que el de intentar arrojar la mayor luz posible que permita abrir al lector, de forma panorámica y propedéutica, a un horizonte temático —en gran parte novedoso, contemplado desde la perspectiva de la pedagogía práctica- en el que se sitúan tres disposiciones y actitudes de fondo insoslayables en todo profesor que aspire a lo mejor de sí en el desempeño de su tarea docente, particularmente en lo concerniente a la relación que está llamado a mantener con sus alumnos. Lo anterior no impide reconocer que existan ya ciertas investigaciones centradas, con más profundidad y amplitud, sobre algunas de esas tres temáticas. Trabajos que posiblemente verán la luz pública de manera específica algún tiempo más tarde que este artículo, que prepara el camino de forma introductoria y global.

Tras esa aclaración formal, entrando ya en materia, es posible asentir convencidamente que los profesores de cualquier nivel no se limitan a instruir a un conjunto de alumnos a su cargo, aun cuando algunos puedan llegar a creer que su tarea tiene principalmente ese cometido. Jackson (19681998), hace ya tiempo, confirmó con suficiente evidencia que, "en" y "a través de" cualquier detalle de su quehacer cotidiano, todos los docentes mantienen ineludiblemente relaciones educativas — sean éstas más o menos plausibles-con sus escolares.

También es cierto que, según las predisposiciones de los diversos profesores, el tipo y la intensidad de las relaciones educativas varían dentro de un continuum que va desde la más notoria frialdad e indiferencia hasta la 
máxima calidez y solicitud a la hora de tratar a los alumnos que constituyen el foco de su atención. En este sentido, el hecho de que tales relaciones tiendan a un polo o a otro es algo de capital importancia dentro del ámbito educativo y, más concretamente, de aquel más propio de la docencia.

El tipo y grado de las relaciones educativas vividas constituyen, ciertamente, un indicador bastante claro de la concepción pedagógica que se tiene de la profesión docente. Cuando, en efecto, el enfoque es eminentemente científico-tecnológico, los profesores son vistos ante todo como gestores y técnicos de la enseñanza, hasta el punto de primar manifiestamente los contenidos, los resultados y la eficacia por encima de la singularidad y del valor personal de los alumnos concretos. Por el contrario, cuando la orientación educativa se centra en la forma en que la experiencia académica y formativa es vivida en cada instante a nivel fundamentalmente humano por ambos protagonistas del proceso educativo, lo prioritario y determinante resulta ser la calidad existente en la relación que se da entre profesores y alumnos con sus nombres propios. La dimensión emocional y ética se ponen en activo, en realidad, sólo en este segundo modo de entender la relación educativa; es decir, cuando se aspira a potenciar, ante todo, el desarrollo humano global de cada alumno singular, sin que ello suponga dejar de favorecer, al mismo tiempo, lo mejor de su progreso académico.

En esta línea, el objetivo de este artículo consiste precisamente en mostrar, por un lado, que los mejores profesores poseen ineludiblemente una serie de disposiciones profundas presentan visiblemente en la relación educativa que mantienen con sus alumnos y en destacar, por otro lado, la influencia que esas actitudes densas ejercen en el desarrollo académico y formativo de los menores a su cargo.

Para este propósito me basaré, aparte de otras fuentes, en tesis pedagógicas centrales de Van Manen - considerado aquí como uno de los más sólidos referentes en la temática que nos ocupa-, así como en ciertos hallazgos de investigaciones en las que he estado involucrado últimamente, que están relacionadas especialmente con la responsabilidad y la esperanza pedagógica de los profesores. Además, por coherencia interna, haré también una serie de observaciones sobre un tópico estrechamente relacionado: el amor pedagógico de los profesores hacia sus alumnos ${ }^{1}$.

Para ser operativo y claro, comenzaré con un presupuesto axiomático del reconocido pedagogo Max van Manen (1998, 78), que confieso hacerlo igualmente mío. Hablando de la relación pedagógica, afirma:

"Las tres condiciones de la pedagogía son las siguientes: el amory afecto, la esperanza y confianza, y la responsabilidad. Porque, ¿es posi- 
ble ser un profesor auténtico sin tener en la actividad educativa una orientación hacia los niños de afecto cariñoso, de esperanza confiada y de responsabilidad?"2.

Esas disposiciones de fondo, como he aclarado más arriba, no entran en contradicción con la posesión de ciertas competencias técnicas (didácticas, organizativas, curriculares, etc.). Con todo, conviene insistir en que, en ausencia de esas condiciones o actitudes profundas, lo más genuino de las relaciones educativas queda fácilmente hipotecado, hasta el punto de llegar a reducirse a una serie de vínculos fríos e instrumentales; algo que reconduce, a su vez, a una concepción de la propia docencia carente de implicación y tendente a la indiferencia. Una cita de Day $(2006,80)$ puede clarificar esa realidad posible:

"No todos los profesores comparten los mismos niveles de compromiso en su trabajo. Para unos, es una parte importante de sus vidas, concediéndole mucha atención y una elevada prioridad. Para otros, sin embargo, la docencia es lamentablemente un mero trabajo. En este último caso, aun cuando pueden a veces desarrollar la enseñanza de manera concienzuda, no constituye el 'centro de gravedad' de su vida docente".

\section{EL AMOR PEDAGÓGICO}

Esta disposición educadora, cuando es asumida hondamente por un profesor, es capaz de alcanzar los estratos más profundos de sus alumnos, porque hace blanco en el núcleo más íntimo y sensible de la persona de cada uno. Por otro lado, ese amor está entrelazado estrechamente en su base y en su dinámica con la "responsabilidad" y la "esperanza" pedagógicas, como se verá fácilmente en adelante.

El amor pedagógico, ciertamente, no se confunde con el amor propio de los padres, los amigos o los enamorados. En primer lugar, porque el profesor no elige a sus alumnos, encontrándose con éstos cuando hacen aparición por primera vez en la clase. En segundo lugar, porque la relación escolar va más allá de aquella estrictamente existencial, al incluir también - por supuesto- la misión de enseñar contenidos académicos diversos. En tercer lugar, porque es un amor fundido con el bien pedagógico de cada alumno particular, siendo así un afecto más específico que el que caracteriza la relación amorosa estrictamente humana. Esto explica que Van Manen $(1998,88)$ haga un comentario como éste: "La relación pedagógica implica siempre una duplicidad de intenciones: el educador auténtico siente, ciertamente, cariño por cada menor confiado por lo que 
es, pero también por lo que puede llegar a ser, de modo que, sin esa duplicidad, la relación deja de ser estrictamente pedagógica”. Afirmación convencida de este autor, que está inspirada sin duda en la concepción sostenida por Bollnow (1979), maestro suyo, cuando aborda la naturaleza peculiar del amor pedagógico. Este gran teórico alemán de la educación, en efecto, aunque admite cierta dosis de eros (amor a los otros que se fija en lo apetecible y valioso de sus vidas, queriéndolos tal como son) y de ágape (amor compasivo imantado por el valor insondable de toda persona, que busca remediar cualquier necesidad de cada una de éstas, y que las estima igualmente con sus debilidades), sostiene que el amor pedagógico se centra en la persona misma de cada alumno, siendo su objetivo transformar al hombre (que anida en cada menor); dicho de otro modo: abrazando tanto sus potencialidades como sus fragilidades, el verdadero profesor llega a pensar para sus adentros respecto a cada uno de sus alumnos: "Te quiero como eres, pero también como lo que aún no eres, y estás llamado a ser"3.

En sintonía con lo dicho más arriba, y esencialmente hablando, el amor pedagógico es también amor auténtico, que incluye ciertamente el afecto y el cariño, aunque no en su versión fácil o laxa. Al hablar de ese amor pedagógico, Van Manen $(1998,80)$ comenta que el primer efecto del mismo es trabar una relación profunda con sus alumnos, citando para ilustrar ese fruto unas palabras de Martín Buber: "la mirada del educador los abraza a todos y los acoge en su recinto más íntimo”. En realidad, lo que quiere destacar con toda claridad el autor es que en toda relación educativa auténtica se da una genuina comunicación ética —en el plano del amor- al centrarse ésta en el núcleo íntimo de la persona del alumno; vínculo que, sin dejar el pensamiento de Van Manen (Ibíd., 87), hace posible que "una parte de la vida del profesor encuentre en ese amor su realización, y que algo similar ocurra con una parte de la vida del alumno".

Sin dejar esa línea, parece conveniente traer a colación también aquí la tesis defendida en el trabajo llevado a cabo sobre esta temática por Wineberg (2006), quien considera que algunos pedagogos — como Kohl (1984), entre otros- se equivocan a menudo cuando reducen cualitativamente el amor a los alumnos por parte de los profesores a un simple amor a tales alumnos en cuanto aprendices. Con profusión de citas y con claridad ética y pedagógica, este investigador sostiene que el amor pedagógico es un amor a la persona misma de cada alumno por su valor intrínseco, que abraza no sólo su dimensión académica sino toda peculiaridad de su personalidad global, en estado de formación ${ }^{4}$.

Esta realidad de hondo calado, sería, por sí misma, objeto de una excelente investigación monográfica. Baste aquí señalar, tan sólo, que en esa 
conexión y comunicación entre lo más valioso de ambas personas —la del profesor y la del alumno- se encuentra la clave de la influencia incalculable que tiene lugar cuando un educador pone en activo el amor pedagógico en su relación con sus alumnos.

Yendo en esta dirección a un plano de mayor concreción, resulta clarividente la observación que hace Day $(2006,33)$ al respecto: "los profesores aman su trabajo - afirma este autor - porque aman a sus alumnos, algo que les insta a buscar constantemente las formas más oportunas de llegar a ellos". Comentario éste penetrante, que encierra en realidad todo un programa de vida para los docentes de cualquier nivel escolar: desde la etapa infantil a la universitaria. Un programa coherente con todo verdadero amor (también con el pedagógico): actuar de forma sumamente creativa e ingeniosa, buscando por todos los medios el verdadero "bien" (educativo en este caso) de aquellos a los que se quiere, y esto tanto en el plano físico y académico como en el psicológico y moral. Se comprende, en esta línea, que Hargreaves encontrara en una interesante investigación que los profesores que destacaban por el cariño y afecto vehiculado en su docencia "se inspiraban en un amplio repertorio de estrategias a fin de llegar a sus alumnos, con el fin de conseguir el máximo de cada uno de ellos; de modo que estos profesores se veían a sí mismos como personas multiestrategas, empleando amplios, variados e inverosímiles formatos de enseñanza y de conexión" (1999, 285289). Sin perder este hilo conductor, resulta perfectamente inteligible que Nieto $(2006,65-82)$ dedique todo un capítulo de su libro a este filón, con el título 'La enseñanza como amor'. En esas páginas, la autora hace ciertos aportes dignos de tenerlos muy en cuenta y de repensarlos para el propósito que nos ocupa. Apuntamos, tan sólo a modo de ejemplo, algunos como los que siguen:

"En algunos ambientes, no queda muy bien hablar sobre la enseñanza y el amor al mismo tiempo. Al fin y al cabo, la docencia es una profesión, como la medicina, el derecho o la ingeniería; quizás por esto pocas veces oímos decir que el amor sea uno de los principales motivos de esas profesiones [...] Pero la docencia es diferente. Ésta implica confianza y respeto, así como unas relaciones cercanas y especiales entre profesores y alumnos. Se trata de una vocación basada en el amor [...] Olvidemos la visión sentimental del amor y pensemos en cómo éste se hace visible a través del trabajo cotidiano de los docentes; porque el amor [pedagógico] no debe verse como una simple concesión emotiva, sino como algo que integra la confianza, la admiración por los alumnos y el aprecio de sus puntos fuertes" (Ibíd., 65-66).

Existen, por supuesto, otros muchos textos que ilustran lo esencial que resulta ser el amor pedagógico en el plano de la relación educativa. Nos 
viene a la mente — entre otros de gran acierto - el que resalta David Hansen, especialmente a través de la actitud de fondo del profesor James, tan real como bien descrito por el autor en su libro, quien personifica ejemplarmente el profundo amor que tiene hacia sus conflictivos alumnos, a los que quiere invariablemente como personas, relativizando en buena medida los contenidos académicos, que el resto de docentes ponen — sin éxito- en el centro de la relación escolar (2001, 85-109).

Sin abandonar el terreno práctico, también ha sido bien abordado el amor pedagógico por Ken Bain, quien trae a colación algunos buenos ejemplos de profesores reales que tratan con ese amor a sus alumnos ya crecidos. La franqueza, delicadeza, apoyo, solicitud, afecto, cercanía y amabilidad, son algunos de los rasgos que adornan el ser y quehacer de los mejores profesores conocidos por este autor. Cito aquí uno de los ejemplos narrados en su libro, que refleja ese trato entrañable tan propio del amor pedagógico en la práctica real y ordinaria:

“Un óptimo profesor de matemáticas tenía un alumno que parecía tener problemas con el cálculo. En concreto, tenía un rendimiento lamentable en los exámenes importantes. Aunque no abandonaba, al final del curso se hizo patente que tenía un serio problema de ansiedad en los exámenes. Un día antes de la prueba final decisiva, el alumno pasó a hablar con su profesor. Este le atendió con amable apertura e inició una conversación informal y distendida con su alumno. Poco a poco fue entrando en materia: ‘Entiendes esto?’, comenzó a preguntarle. Viendo que el alumno iba respondiendo adecuadamente a sus cuestiones, el profesor le invitó afablemente a que le explicara conceptos de la materia y a que hiciera problemas más bien difíciles. Pasó así más de dos horas con él, apoyando cálidamente sus explicaciones y soluciones. Comprobó, con satisfacción, que su alumno sabía mucho más que lo que él podía imaginar a raíz de las notas tan bajas de los exámenes que ya había hecho con anterioridad. Después de esa larga tutoría improvisada, el profesor miró a su alumno con un tono de clara complicidad afectiva, y le dijo: 'Acabas de hacer un buen examen oral de cálculo. He de pensar aún la nota exacta, pero has aprobado el curso'. El alumno, asombrado, le preguntó qué debía hacer con el examen final del día siguiente. El profesor le respondió sonriendo: ‘Ah, no sé! ¿Por qué no vas y lo haces tan sólo por gusto?’. El alumno fue al examen y obtuvo un excelente” (2006, 145-146).

No cabe duda que esa tipología de relaciones, tan afables y acogedoras a nivel de trato personal como exitosas en el plano académico, tienen su raíz originaria en un amor pedagógico por los alumnos, que impulsa al profesor a buscar las formas más oportunas de llegar a todos los que le han sido con- 
fiados, con el fin de encontrar creativamente todos los medios posibles que faciliten el desarrollo escolar y humano de esos alumnos, a quienes lleva en su interior con profundo afecto y entrañable estima.

Podrían añadirse aquí algunos comentarios más en torno a la condición esencial del amor pedagógico en los docentes de todos nuestros centros educativos, que ejercen su tarea en medio de situaciones a menudo difíciles de abordar en el caso de carecer de actitudes que promuevan relaciones educativas de ese corte. Con todo, unas líneas de un magnífico profesor, reconocido públicamente por su amor comprobado a su profesión y a sus alumnos, pueden finalizar mejor y con mayor propiedad este primer apartado de la triada tematizada:

"Los profesores han de querer de verdad, y se les ha de notar. Y querer de verdad, todos sabemos de qué va la cosa. Estamos en el campo de la pedagogía del amor. La gratuidad y la incondicionalidad son dos atributos que no están demasiado de moda, que ni cotizan en bolsa, ni fisgonean en ninguna resolución administrativa. Sobre ello tampoco he sabido encontrar ninguna cita en la nueva Ley de Educación. Es cierto que hay una referencia clara a la importancia de la educación emocional, pero parece que hablar de amor no sea pedagógicamente correcto.

Sí, los profesores han de querer de verdad, y se les ha de notar. Dicho en otras palabras: cuando un niño o una niña pone los pies en la escuela ha de tener la garantía de que entra en un espacio de relaciones personales donde es querido por los docentes que le atienden, por ser quien es, por su valor personal, tanto si lleva las manos limpias como si no, si va peinado con raya a la derecha como si aparece con una cresta iridiscente. Los chicos y chicas saben perfectamente quién los quiere o los ha querido; quién vela por no pasar del cumplimiento estricto y literal de los deberes establecidos administrativamente y quién se entrega generosamente a la empatía, a la simpatía y a la benevolencia más comprometida [...] ¿Os habéis sentido queridos así por vuestros profesores o profesoras?” (Poch, 2008, 12-13).

La cita es fecunda en temas ético-pedagógicos de bulto, constituyendo una invitación a pensar en la necesidad imperiosa de cómo poder conseguir tener en nuestros contextos educativos profesores con un talante así. Sin entrar aquí más en detalle, nos podemos quedar con la interpelación encerrada en la pregunta última de ese comentario, adoptando una actitud tan positiva como retadora. 


\section{LA RESPONSABILIDAD PEDAGÓGICA}

Pasamos ahora a la segunda condición esencial para que los profesores sean verdaderamente tales, situados siempre en el plano de la relación educativa con sus alumnos. Aunque la temática de la responsabilidad pedagógica ha sido objeto de una investigación concreta por mi parte, en este estudio - de índole panorámica - sólo será abordada de forma delimitada, dado que la finalidad de tratarla en este apartado es necesariamente comedida. Hecha esa observación formal, en primer lugar intentaré clarificar la trascendencia que tiene esa actitud medular en los alumnos cuando es asumida realmente por profesores que son verdaderamente comprometidos. En segundo lugar, daré a conocer de manera sucinta el proceso metodológico fenomenológico-hermenéutico aplicado en la investigación realizada sobre el tema de la responsabilidad pedagógica de los profesores, pasando después a considerar alguno de los hallazgos significativos de dicho trabajo con el fin de ilustrar brevemente la naturaleza e influencia de esa disposición esencial en todo buen profesor. Siguiendo esta lógica, dividimos en dos el contenido de este segundo apartado.

\subsection{Aproximación teórica al tema de la responsabilidad pedagógica}

Para este fin, elijo nuevamente a Van Manen como autor de partida para iniciar, a modo de marco global, los comentarios pertinentes sobre esta temática. Este autor holandés, de casi obligada referencia, inspirándose con fuerza en las tesis principales de Lévinas, es sin duda uno de los que más claramente ha traducido al campo de la 'pedagogía práctica y vivida' las ideas-fuerza del reconocido filósofo lituano-francés sobre el tema de la responsabilidad — pedagógica - en muy diversas publicaciones suyas. Veamos aquí tan sólo algunas de sus reflexiones al respecto:

"Hay algo en los pequeños que nos estimula y nos atrae hacia ellos de forma muy concreta [...] Esa inclinación pedagógica es la que nos llama, la que nos emplaza a escuchar sus múltiples necesidades" (1998, 41-42). "Podríamos decir que la debilidad o vulnerabilidad del niño se convierte en una curiosa fuerza sobre el adulto [...] Por supuesto, cabe la reacción de ignorar simplemente al niño y seguir con nuestros asuntos, como si no tuviéramos responsabilidad pedagógica. ¿Por qué tengo que escuchar a un niño? ¿Qué tengo que ver con la vida de este niño? De hecho, lo que hacen algunos es ignorarlos. Pero, para otros, desatender el sentimiento de responsabilidad no les es sencillamente posible. Un niño me pide ayuda, y siento que debo actuar de forma receptiva y responsable con él. 'Sentirse reclamado' es lo más importante, el significado más profundo de ser educador o pedagogo” (Ibíd., 109) 
Cualquier alumno, por tanto, se convierte en Otro que entra con su llamada en la vida de todo profesor implicado, y al irrumpir así en su ser se convierte en "su" alumno, no en "uno" más de la masa de la clase. El verdadero profesor — no el simple técnico o el asalariado de la enseñanza — capta con fina sensibilidad las continuas necesidades de todos y cada uno de "sus" alumnos y se ve impelido a responderlas haciéndose cargo de cada uno de ellos. De esta suerte, nace en él la responsabilidad pedagógica, de modo que los reclamos de todos los niños o jóvenes que le han sido encomendados encuentran eco en lo más íntimo de su ser, resultándole imposible así ignorarlos, es decir, adoptar una actitud superficial de indiferencia. En esta línea, el bien pedagógico de cada menor a su cargo se convierte, aun presentándose usualmente en formatos múltiples de vulnerabilidad, en una fuerza que no puede dejar de recibir de una forma incluso conmovedora. Avancemos un poco más junto con Van Manen.

"En realidad, no experimento la subjetividad del otro hasta que no logro superar esa centralidad de mi self en el mundo [...] La vulnerabilidad del otro es el punto débil en el blindaje del mundo centralizado en mí mismo. Cuando veo a un pequeño necesitado o herido de algún modo, noto la necesidad de olvidarme de mis preocupaciones actuales. Ya no tiendo a dejarme llevar por mi agenda personal; porque debo estar ahí, para esa otra persona [...] Por tanto, cuando un niño está mal, y yo realmente 'veo' a ese niño en su vulnerabilidad, entonces estoy en posición de hacer algo por ép”. [Más aún], "siento al otro como una 'voz', como una 'llamada'. Precisamente esto es lo que queremos decir cuando hablamos de nuestra vida con los pequeños entendida como una vocación, como una llamada [...]

En este sentido, el tacto pedagógico es la práctica de estar orientados hacia los otros". [Dicho de otro modo]: "el verdadero tacto está movido por el amor y por la inclinación hacia el Otro, más que inclinado hacia el propio self, no moviéndose por intereses egocéntricos, tendentes a convertir a los Otros en apéndices de uno mismo” (Ibíd., 147-153).

Lo apuntado por Van Manen nos hace pensar en que la responsabilidad pedagógica es, por esencia, rotundamente exigente, en cuanto insta al profesor comprometido a salir del propio recinto egocéntrico y, desde esa disposición, des-vivirse hasta el punto de olvidarse totalmente de la propia agenda personal-profesional, para llegar a vivir en y para los Otros.

Por otro lado, la responsabilidad pedagógica implica una sensibilidad penetrante y viva, capaz de captar las manifestaciones propias de la vulnerabilidad de los alumnos; esto es, las necesidades más íntimas y silenciosas 
de éstos en el contínuum de la vida en las aulas; sensibilidad que da un tono exquisito al clima de la clase, a la urdimbre de relaciones que mantiene el profesor con sus alumnos. Sigamos de nuevo a este autor sin dejar esta veta:

"Un profesor de verdad 'sabe cómo ver' a los niños: se percata de la timidez de uno, del bajo ánimo de otro, de los deseos o expectativas de un tercero. Para ver de este modo se necesita algo más que ojos; se precisa tener un sentido de responsabilidad [...] De una forma u otra, el profesor comprometido con los niños hace el esfuerzo de 'tocar' a cada alumno. ¡Qué fácil es, si no, dejar que pasen los días sin estar jamás en contacto con determinados niños! El niño callado, el niño 'fácil', puede quedarse sin ese contacto durante mucho tiempo [...] Por su parte, los más rechazados y marginados lanzan 'llamadas' todavía más agudas para que se les preste más atención [...] No son muchos, desafortunadamente, los profesores que entran de verdad en la 'casa' de cada uno de sus alumnos" (Van Manen, 2008, 39-40).

En los comentarios anteriores hay mucho contenido temático digno de glosar, profundizar y matizar en torno al asunto sumamente rico que nos ocupa: la responsabilidad pedagógica. El mismo Van Manen, ha abordado y clarificado con más reposo ciertos puntos abordados más arriba en otros escritos suyos de notorio interés ${ }^{5}$. También en nuestro contexto próximo hay autores que han focalizado su atención en una dirección muy próxima. Vienen a la mente, enseguida y por ejemplo, los profesores Bárcena, Mèlich, Mínguez y Ortega. Imposible recoger en este tan acotado espacio sus finas y numerosas apreciaciones en este campo. Con todo, ¿cómo no citar aquí, con una intención tan sólo ilustrativa, algunos de sus comentarios más vinculados con la temática tratada? En un excelente libro conjunto, los dos primeros autores comentan con acierto:

"La traducción pedagógica del planteamiento de Lévinas nos la ofrece Max Van Manen en su libro El tacto en la enseñanza. Escribe ahí el pedagogo holandés: "El adulto es sensible a la vulnerabilidad o necesidad del pequeño, y experimenta una extraña sensación: la verdadera autoridad en ese encuentro está en el niño, no en el adulto [...] La debilidad del pequeño se convierte en una curiosa fuerza [moral] sobre él [...] La autoridad pedagógica la concede el niño, produciéndose en un encuentro que el adulto experimenta a través de la responsabilidad que tiene del niño" (Barcena y Mèlich, 2000, 141).

Curiosamente, el pequeño - con toda su indigencia y falta de poderes quien ofrece la autoridad pedagógica a quien lo educa en forma de reclamo ético: 'Hazte cargo de mí, ayúdame a sacar lo mejor de mí mismo; dame tu 
tiempo, que es para mí; dame a ti mismo, respóndiéndome con tu amor' (Cf. Mèlich et al., 2001, 33).

En estrecha conexión de ideas se expresan Ortega y Mínguez $(2003,48)$ cuando se refieren a lo esencial de la tarea educadora, citando a menudo, entre otros, a Van Manen:

"Educar no es sólo enseñar, y enseñar bien. En el núcleo del acto educativo hay siempre un componente ético, una relación ética que liga a educador y educando y que se traduce en una actitud de acogida y de compromiso, en una conducta moral de hacerse cargo del otro. Es esta relación ética, responsable, la que define y constituye como tal a la acción educativa”.

Al decir de estos autores, educar es hacer nacer "alguien nuevo"; una tarea esencialmente ética que - al surgir y acabar fuera de uno mismo- supera todo asomo de reproducción o prolongación del "yo egocéntrico" de quien la lleva a cabo. Una tarea en donde el protagonista es, en realidad y continuadamente, el Otro. De ahí que, a diferencia del paradigma convencional imperante, ha llegado la hora de dar el salto a una genuina pedagogía de la alteridad, como la propone y comenta con finura exquisita Ortega (2004) en un artículo claro y profundo, que aborda las dos caras de la misma actitud profunda de todo educador coherente con su misión: acoger a los menores, por amor a su insondable valor personal, y responder de ellos ilimitadamente con toda su concreción humana, en el fondo por el mismo motivo.

En realidad, lo dicho hasta aquí constituye sólo una seria invitación a seguir profundizando en este venero descubierto -relativamente- no hace mucho tiempo, rico en tesis y propuestas de valor incalculable para una nueva orientación de la pedagogía teórica y, particularmente, de la pedagogía práctica, cotidiana, vivida.

\subsection{Breve aportación ilustrativa desde una investigación sobre este tema}

Antes de mostrar esa ejemplificación, lo más razonable es intentar delinear aquí, aunque sea a grandes rasgos, el proceso metodológico seguido en la investigación llevada a cabo sobre el tema que nos ocupa; esto es, la "responsabilidad pedagógica de los profesores a partir de su experiencia vivida".

La metodología utilizada ha sido la fenomenológica-hermenéutica ( FH); y, en concreto, la versión teórica-práctica que propone Van Manen, principalmente en su libro Investigación educativa y experiencia vivida ${ }^{6}$. El objetivo 
siempre presente a lo largo de esa investigación ha sido intentar aprehender y captar la esencia de lo que "es" - y, por lo mismo, de lo que "debe ser"- la "responsabilidad pedagógica" de los buenos profesores en relación a la atención, solicitud, dedicación y preocupación que, en todos los órdenes de la relación educativa, están llamados a asumir y practicar con sus alumnos.

La "muestra", para entendernos aquí, ha estado formada por unos 50 profesores de ambos sexos, de todos los niveles del sistema educativo - desde infantil a universidad-y de diferentes lugares de nuestra geografía española. Esos profesores han sido elegidos atendiendo a un criterio práctico: su excelente reconocimiento por parte de directores, colegas, padres, menores implicados, y también por la comunidad educativa más amplia (en cinco casos galardonados públicamente), gracias a su demostrada "preocupación” y probado "compromiso" expresados en su —sencilla pero admirable- forma de vivir "volcados" en sus alumnos en el ejercicio de su tarea docente y educadora. Todos ellos fueron invitados a colaborar en una investigación $F H$, dándoles ciertas pistas sobre el modo de narrar -en formato fenomenológico- "relatos anecdóticos" de una página y media de extensión aproximadamente, con el fin de recoger alguna/s de sus "experiencias vividas en relación a su propia responsabilidad con algunos de sus alumnos". Cada uno de ellos escribió uno o dos "relatos"; uno de los cuales presentado enseguida, tras estas páginas introductorias.

a) Recoger la experiencia vivida, a través precisamente de esa modalidad escrita comentada, ha sido la primera fase la aplicación del método FH. En este sentido, puede decirse que el conjunto de todos esos "relatos anecdóticos" han constituido la "masa de datos", que ha sido trabajada en las fases siguientes.

b) Reflexionar sobre dicha experiencia vivida ha constituido la segunda fase. El cometido principal de esta etapa ha sido el análisis temático, que se ha hecho en dos planos: el primero, realizando la denominada reflexión macro-temática (buscando una frase sentenciosa para cada uno de los relatos, con la intención de captar tentativamente el significado potencialmente esencial del fenómeno investigado, esto es, la responsabilidad pedagógica de los profesores); el segundo, llevando a cabo la llamada reflexión micro-temática (seleccionando frases clave de cada relato, con la intención de aferrar lo más atinadamente posible los significados esenciales implícitos en esa experiencia vivida por los profesores de forma más matizada) (Van Manen, 2003, 108-113).

c) Realizar la 'reducción eidética' sobre ese conjunto de frases y primeros ensayos de redactar párrafos sensibles fenomenológicamente (a modo de borradores provisionales) en torno a posibles significados esenciales sobre el 
tópico estudiado, ha constituido la tercera fase. En ese momento del proceso, se ha tratado de aplicar el método de la variación imaginativa (Ibid., 125) para probar - $\mathrm{O}$ no- si unos presuntos significados esenciales (o algunos relatos anecdóticos completos) iban siendo significativos realmente, o sólo aparentemente, en relación a la buscada esencia de la verdadera responsabilidad pedagógica. A modo de ejemplo, se ha dado algún caso que, sin dejar de ser valioso el relato mismo escrito por un profesor excelente, ha sido descartado por caer en la cuenta, mediante esta forma de proceder, que la narración concreta manifestaba más bien una sincera "buena voluntad" de hacer cosas por los alumnos que una "responsabilidad pedagógica" real, en el sentido que Lévinas, Van Manen y otros autores parecen apuntar, también en este estudio.

d) Escribir reflexivamente acerca de la experiencia vivida ha conformado la cuarta y última fase de la investigación. Lo que aquí se ha hecho es escribir el $T F$ sobre dimensiones que evocan la esencia del fenómeno investigado: de la responsabilidad pedagógica de los profesores. Esta es la etapa del proceso más crucial y menos fácil, por la sensibilidad y la calidad del lenguaje escrito formal que requiere la elaboración del $T F$, que, a juicio de Van Manen (Ibid., 27), "constituye el principal objetivo de este tipo de investigaciones". Tomado en su globalidad, el TF escrito en nuestra investigación fenomenológica-hermenéutica está formado por medio centenar de páginas en las que se han recogido los hallazgos esenciales de la experiencia vivida investigada: en este caso, de la vivenciada por cincuenta profesores realmente comprometidos con su docencia y sus alumnos, a partir de sus "relatos anecdóticos" en los que narran sus propias experiencias de responsabilidad pedagógica en su relación mantenida con los alumnos en diversos momentos significativos del mundo de la vida educativa cotidiana. A diferencia de una investigación cualitativa convencional, dicho $T F$ no se confunde ni con los usuales "análisis de datos" ni con las habituales "conclusiones finales" que suelen espigar los resultados más interesantes de los convencionales trabajos, incluso de los más cualitativos a nivel metodológico y los más humanistas a nivel temático. Esta precisión es de gran importancia dado que, en la investigación que nos ocupa, la aspiración del TF escapa a todo intento de explicación causal para centrarse, tan sólo y con la máxima puridad, en aprehender lo genuinamente esencial del fenómeno estudiado: desde la naturaleza de la responsabilidad pedagógica hasta su sentido en el marco global de la tarea educadora, pasando por la influencia de esa responsabilidad en la vida académica y personal de los alumnos. Ahora bien, como apunta Van Manen, el reto en este momento consiste en intentar lo más felizmente posible "transformar la experiencia vivida de forma pre-reflexiva - de la responsabilidad pedagógica en este caso- en un texto (fenomenológico) que exprese su esencia, de tal modo que el efecto del texto sea, a la vez, un revivir reflejo y una aproximación reflexiva del significado de tal fenómeno: una noción por la 
cual el lector pueda cobrar vida con fuerza en su experiencia vivida" (Ibid., 45). Para ello se ha puesto todo el cuidado posible, a la hora de escribirlo, en aunar las dimensiones cognitiva y pática, conceptual y poética, informativa y evocadora, de forma que los contenidos más prosaicos estén en oportuna armonía con los literarios, vivos y expresivos.

Dada la extensión limitada de este estudio, me centraré a continuación en ilustrar una discreta muestra de la riqueza de la responsabilidad pedagógica a partir de alguno de los "relatos anecdóticos" propios de la investigación realizada recientemente sobre esta temática. Para ello, a modo de sobrio ejemplo, transcribo unas breves páginas entresacadas de lo que, en la metodología empleada, constituye el "texto fenomenológico" (TF).

"Hace poco fui profesor-tutor de un grupo de alumnos de cuarto de la ESO. Era, para decirlo de una forma fácil de entender para la mayoría de los profesores de cualquier centro docente, 'el grupo difícil'. Alumnos con visibles dificultades de aprendizaje y algunos con serios problemas conductuales. Uno de estos alumnos era Soufiane, alumno magrebí, repetidor en tercero de la ESO, y que al tener ya los 16 años podía finalizar la educación obligatoria, si así lo decidía.

Durante los primeros dos meses del curso, el comportamiento de Soufiane no había sido del todo correcto (había tenido problemas con más de un profesor). Se mostraba distante, chulesco y con pocas ganas de trabajar y sacarse el graduado escolar (objetivo principal para este grupo-clase). Aunque logré hablar con él en dos o tres ocasiones en las denominadas tutorías individuales, intentando así conocer la razón de su comportamiento y de su desgana a la hora de estudiar, no obtuve ningún resultado positivo de esos encuentros. Ni una palabra.

Un día por la tarde, sin embargo, Soufiane vio cómo yo marchaba tarde del instituto, mientras él acababa de jugar un partido de fútbol con otros compañeros. Con tono chulesco me dijo: 'Profe, ¿me llevas a casa en coche o qué?'. Sin pensarlo dos veces, le dije: 'Ok, sube; ¿dónde vives?'. Soufiane vivía en la otra punta de la ciudad (unos 45 minutos a pie) y quizás en ese nuevo clima de proximidad y confianza que afloraba en esa ocasión (él nunca habría imaginado que le quisiera llevar a su casa en coche) abrimos, tras unos minutos de crear clima, el tema de su comportamiento y trabajo escolar. En aquel momento, el alumno 'se abrió'. Me dijo que no tenía ganas de hacer nada porque en su casa no le valoraban nada de nada; que su padre tan sólo se dirigía a él para darle alguna bronca, que no lo tenía en cuenta para nada y que no se interesaba por él (‘'no sabe ni qué curso hago!’), y que todo eso le desmotivaba mucho. 'Una vez llego a casa —me dijo-, 
¿qué ganas tengo así para abrir la libreta y ponerme a estudiar? ¿Para quién? ¿Quién me lo valorará?’. Todo eso me lo decía con lágrimas en los ojos. Se sentía impotente. Le toque la espalda y le estimulé con el fin de que viera que había de trabajar para él, que el graduado escolar le permitiría abrir puertas laborales y educativas. Le propuse que los viernes -el único día que comenzamos a las nueve de la mañana en el instituto- podía venir una hora antes y que me tendría disponible para responderle todas las dudas; que podría contar conmigo. Que yo, de la misma manera que le daría 'un toque' cuando su comportamiento no fuera el correcto y su trabajo escolar bajo, le daría un fuerte abrazo cuando viera que su comportamiento y su trabajo mejorase.

De camino hacia casa pensaba yo: '¡Fíjate. El muchacho más 'duro' del grupo-clase, en el clima de intimidad y confianza que se ha dado al ofrecerle llevarlo a casa, me ha comentado con todo detalle sus miedos y ansiedades. Si mañana viene al instituto a las 8 -aquel día era jueves - saldrá adelante; porque acudir de forma voluntaria a trabajar a las 8 de la mañana, y viniendo caminando 45 minutos desde su casa, significa que se habrá de levantar a las 7... o antes!'.

La verdad es que esa mañana del viernes llegué con nervios al instituto, esperando poderlo ver en clase. Al entrar en el instituto, el conserje me dijo: '¿Has castigado a Soufiane? Te está esperando arriba'. Después de ver estas reacciones de Soufiane, me quedé pensando conmovido: 'iQué necesidad tan grande tienen los alumnos más desvalidos de mi disponibilidad incondicional!’”.

(Profesor de cuarto curso de la ESO)

Este profesor, como otros muchos, tiene ante sí una realidad no muy risueña. Da clases al "grupo difícil" de ese último curso de la ESO. Uno de los que más destaca es un alumno magrebí, distante, chulo, indiferente ante su escolaridad. Otro colega, en su lugar, posiblemente hubiera ignorado a ese chico, evitando todo enredo y limitándose tan sólo a instruir bajo el fácil lema: “Sálvese quien pueda!”.

Pero nuestro profesor parece no resignarse a una postura de mínimos. Tiene asumida una innegable responsabilidad pedagógica: ¡Le importa, y mucho, "su" alumno Soufiane! ¡Siente en su interior una llamada aguda y suplicante que le reclama ayuda de forma lastimera y silenciosa!

De hecho - como seguramente con otros necesitados- ya ha hecho algunos intentos de introducirse en el mundo secreto de esa "oveja negra" a través de varias tutorías individuales; y el hecho de que ese primer ensayo de 
responsabilidad no haya llegado a buen fin, no parece que haya introducido en él la tentación de rendirse a probar otras iniciativas más logradas. En realidad, de forma prerreflexiva, nuestro profesor ha hecho muy suya la propuesta de Lévinas, que parece susurrarle al oído: "sé responsable del otro, sin esperar la recíproca, aunque ello te cueste la vida; la recíproca es asunto suyo" (1991, 93). Además, como si asintiera a lo que el filósofo lituano pareciera instarle, entiende que "nadie puede decir jamás: he cumplido todo mi deber... salvo el hipócrita" (Ibid., 98). Nada, en efecto, más lejos de él que traicionar su impulso de donación a su alumno Soufiane. Sin dejar de cejar en su compromiso, desea —más bien— seguir al acecho de otra posibilidad más feliz. ¡Y esa ocasión llega!?

Es fácil imaginarse al profesor sonriendo feliz para sus adentros cuando, con chulería, Soufiane, cansado después de un agotador partido de fútbol, al cruzarse con el coche de su profesor - saliendo despacio, con la actitud de quien espera lo inverosímil - le lanza la provocadora pregunta: "Profe, ¿me llevas a casa en coche o qué?". Como él mismo relata, sin pensarlo dos veces, le dice: "Ok, sube; ¿dónde vives?". Vislumbra la oportunidad de que el muchacho se abra en un clima distendido. Se lo dice su sensibilidad y su responsabilidad pedagógica, que preceden a todo pensamiento reflejo. Y es que, cuando se es realmente comprometido y se vive, hasta desvivirse, para cualquier Otro, la intuición y el impulso éticos preceden al pensamiento y a la libertad cimentados en el razonamiento. Con seguridad, ese profesor - como afirma Lévinas - "no toma conciencia de esa necesidad de responder como si se tratara de una obligación o un deber particular sobre el que tiene que decidir. Es, en realidad, la presencia del Otro la que [le] emplaza a responder de él [de su alumno]; [puesto que] el Yo, ante el Otro, es infinitamente responsable" (Lévinas, 1993, 47).

Es en ese clima acogedor cuando ese profesor palpa aún de manera más cruda la vulnerabilidad de Soufiane, su apurada y cruda vida personal, familiar y escolar; atmósfera acogedora hasta el punto de invitar a éste a contarle toda su problemática interna "con lágrimas en los ojos"... porque se sentía impotente.

Una segunda tanda de "llamadas" sacude las entrañas del profesor a partir de ese momento. Con más lucidez que al comienzo, siente que ha de hacerse cargo de su menesteroso alumno. Olvidado ahora del propio tiempo y persona, se ofrece con total disponibilidad a ayudar con todo su ser a su alumno, acariciando su bien personal y pedagógico: “iQuizás con mi apoyo llegue a dar de si lo mejor!".

Con esa disposición responsable inicia su conmovedor diálogo con su alumno, que termina en la propuesta final del relato: quedar a su disposición 
para sacarlo de su pozo hondo y oscuro, esperando tocar así las fibras íntimas de la propia responsabilidad de su muchacho necesitado. Actitud ésta que también parece haberle susurrado en voz baja de nuevo Lévinas: [Tú eres] "responsable con una responsabilidad total, que responde de todos los Otros [también de Soufiane] y de todo lo de los Otros, incluida su misma responsabilidad" (1991, 93). Como reanimado con esa actitud ética-pedagógica que le exige todo de él en búsqueda del bien personal y pedagógico de su alumno, al volver a casa ya solo en su coche, el profesor va musitando en sus adentros: si accede a mi apoyo "saldrá adelante".

Sencillo, pero bonito ejemplo - entre otros muchos- del significado e influencia de la responsabilidad pedagógica cuando la poseen vital y profesionalmente los profesores y la ponen en activo: “ $i$ Hasta los más duros y difíciles pueden son tocados, facilitando así su cambio!".

\section{LA ESPERANZA PEDAGÓGICA}

Las expectativas, tal como usualmente han sido estudiadas y aplicadas, pertenecen al ámbito académico y son de orden psicológico. La esperanza pedagógica, aunque en ocasiones utilice las expectativas positivas, es un fenómeno perteneciente por esencia al plano pedagógico en su sentido más hondo. Un comentario de Van Manen aclara esto algo mejor:

"Depositar esperanza es más una forma de estar presente para él que un modo de hacer las cosas. En realidad, es un modo de ser. En el día a día, abrigamos muchas expectativas y deseos: espero que este alumno mejore su caligrafía, que el otro no se aficione a las drogas, etc. Ahora bien, estas esperas u otras van y vienen con el paso del tiempo. Lo importante, sin embargo, es concebir la relación educativa diaria con esperanza $(1998,81)$.

En otro lugar, el mismo autor clarifica que sólo el amor es capaz de generar una confiada y sólida esperanza pedagógica en un educador, comprometiéndolo a fondo con el menor a su cargo, de quien se responsabiliza incondicionalmente clavando su mirada en su valor personal cuasi infinito. ¡Una actitud que conmueve las entrañas de los alumnos más impermeables!

La interpretación pedagógica de la esperanza deja claro que sólo podemos depositarla en los niños o jóvenes a los que de verdad amamos, no en sentido sentimental, sino en la acepción más pedagógica. Así entendida, la esperanza nos urge a pensar de cada uno de ellos: "No te dejaré tirado; estoy convencido de que puedes llegar a ser más y mejor" (Ibidem). 
Y continúa: "La esperanza se nutre de la paciencia, la apertura, la creencia y la confianza en las posibilidades de nuestros menores. Cuando ellos perciben nuestra confianza se ven animados a confiar en sí mismos. La confianza les hace capaces" (Ibídem).

Es decir, cuando un educador derrocha incansablemente auténtica esperanza en sus alumnos, hasta el punto de lograr que sea sentida vivamente por ellos, prácticamente nada puede impedir que, antes o después, consiga rectificar la miope visión que tienen de sí mismos, cambiando esa imagen vacilante de su valía académica y humana por otra cimentada en una animosa confianza en la capacidad latente en los recónditos estratos de su interioridad.

Por otra parte, "la esperanza pedagógica es nuestra experiencia afianzada de las posibilidades de desarrollo de los niños y jóvenes. Es una esperanza que sigue confiando con firmeza a pesar de las veces que nos hayan podido poner a prueba" (Ibíd, 82).

Comentario éste que delinea con bastante acierto algunos de los rasgos medulares de la esperanza pedagógica. Permaneciendo en esa línea, puede decirse también que la esperanza pedagógica va más allá de una ligera postura 'optimista', al ser cabalmente realista, alejándose así al mismo tiempo del sombrío pesimismo. El profesor esperanzado, ciertamente, no se queda paralizado mirando el perfil brumoso de la realidad: "los chicos no tienen interés, no se esfuerzan; son difíciles, muy diversos, muchos, y no responden fácilmente; los recursos son escasos; las familias no colaboran y la administración tampoco como debiera". La esperanza no ignora el hecho de que todos los handicap pensables condicionan la tarea educativa con sus alumnos, pero los franquea sin dejarse determinar por ellos. O dicho con otras palabras: la esperanza pedagógica de un profesor, cuando es realmente firme, es capaz de ver más allá de toda dificultad aparentemente insalvable para clavar su mirada confiadamente en la vertiente luminosa de la realidad; esto es, en las cualidades, las señales, los deseos y, sobre todo, en las inmensas potencialidades encerradas en lo más hondo de los alumnos que tiene a su cargo. Porque, ¿quién puede, a priori, poner límites a la inmensa capacidad escondida en cada uno de sus alumnos? ¿Quién es capaz de calcular el alcance de la influencia que suscita un educador esperanzado en ellos?8.

De forma más concreta, esas cualidades de la esperanza se ven en casos evidentes que confirman el potente influjo que tiene esa disposición capital en los menores en formación. La mente se detiene enseguida en el ejemplo de la vida de Hellen Keller: la niña sorda, ciega y muda, que se transformó gracias a la esperanza exigente, paciente y constante de su educadora Anne Sullivan, de forma que de una muchacha incontrolable pasó a 
ser una persona sobremanera extraordinaria a nivel intelectual y humano. Palomero et al. (2009) realizan comentarios de gran interés a partir de esta figura. Fijémonos, por ejemplo, en éste:

"Como decía Freire, el problema no son las dificultades, sino cómo transformar éstas en posibilidades; la historia de Hellen Keller nos muestra el poder de la educación en orden a la superación de limitaciones y barreras, la capacidad transformadora de la pedagogía de la esperanza" $(2009,17)$

Los profesores realmente esperanzados no se dejan cegar por las nebulosas apariencias que se presentan a la vista en primer plano, sino que intuyen que su protagonismo puede dar la vuelta a la realidad menos pensable. ¿No es esto lo que nos confirma, con sobrada experiencia, Daniel Pennac a lo largo de su conocida novela autobiográfica?

"A todos los que hoy imputan el fracaso y la constitución de las bandas sólo al fenómeno de los suburbios, les digo: tenéis razón; sí, el paro; sí, la concentración de los excluidos; sí, las agrupaciones étnicas; sí, las tiranías de las marcas; sí, las familias monoparentales; sí, el desarrollo de una economía paralela y los chanchullos de todo tipo; sí, sí, sí... Pero guardémonos mucho de subestimar lo único sobre lo que podemos actuar personalmente, y que además data de la noche de los tiempos pedagógicos: la soledad y la vergüenza del alumno que no comprende. Sólo nosotros podemos sacarlo de aquella cárcel, estemos más o menos formados para ello" $(2008,36)$

Así es. Pueden abundar condiciones externas indeseables, como las que apunta el autor en su comentario, o como las que cada profesor también conoce de cerca en sus circunstancias sociales, familiares y escolares de tintes más que oscuros. Pueden escasear los resultados positivos inmediatos, como las reacciones deseables y razonables a nivel académico o conductual, por parte de un buen número de alumnos. Pero, sólo nosotros somos los que no podemos faltar. Sólo nosotros somos los que no les podemos fallar. Sólo nosotros somos los que debemos permanecer fielmente y siempre a su lado, esperando las ocasiones oportunas para avistar oportunidades de oro que nos permitan entrar en los entresijos de las vidas de esos menores a nosotros confiados, para ser así sensibles a las agudas llamadas que tan a menudo lanzan reclamando nuestra ayuda aun sin saberlo ellos mismos, que sólo nuestro amor esperanzado es capaz de captar y secundar. Eso es lo que, en el fondo, suscriben las convencidas palabras de Pennac, angustiado durante años en su vivida experiencia escolar: "Basta un profesor — i uno solo! — para salvarnos de nosotros mismos” (Ibíd., 219). 
¡Qué realista fue con sus alumnos después este personaje francés, una vez convertido en profesor!

"La mayoría de mis alumnos eran como fui yo mismo, niños y adolescentes con dificultades escolares más o menos grandes. Los más afectados presentaban poco más o menos los mismos síntomas que yo a su edad: pérdida de confianza en uno mismo, renuncia a cualquier esfuerzo, incapacidad para concentrarse, dispersión, mitomanía, constitución de bandas, alcohol a veces, drogas supuestamente blandas también. Pero, eran mis alumnos (un posesivo que no indica propiedad, sino conciencia de mi responsabilidad de profesor comprometido con ellos). Parte de mi oficio consistía en convencer a mis alumnos más abandonados (por ellos mismos) de que [...] el día y la hora de entrega de un ejercicio no son negociables, de que unos deberes hechos de cualquier manera deben repetirse para el día siguiente. Pero, sobre todo, de que nunca, jamás de los jamases los dejaría en la cuneta" (Ibíd., 143).

El trabajo diario de Pennac con sus alumnos debió ser, a buen seguro, realmente duro. En sus palabras se vislumbra que, en su lucha por sacar del fango a esos muchachos enfrascados en formas de ser y de hacer que los abocaba a un fracaso escolar-personal más que probable ${ }^{9}$, necesitaba adoptar una actitud tan afectiva como exigente. Talante éste sólo comprensible por estar anclado en la esperanza pedagógica que le sostenía en la relación con sus alumnos (tirados por la vida, y quizás por otros profesores); a los que había prometido fielmente que... "inunca, jamás de los jamases, los dejaría en la cuneta!”. Una exigencia, en fin, basada en una esperanza realista. Porque dejar de plantear metas alcanzables a su medida, con tanto esfuerzo como estímulo confiado, ¿no implicaría decirles, en el fondo, de forma indirecta: "Finalmente, he dejado de esperar ya en vosotros"?

Basten aquí dos citas para poner fin a un tema, en realidad, todavía poco explorado. Las dos quieren incidir en la visión positiva que implica para los profesores tener esperanza pedagógica.

"No faltan quienes comentan que, con independencia de lo buenos que sean los profesores, sólo pueden ayudar a escapar a su situación desfavorable a una minoría de alumnos de entornos socioeconómicos y emocionales deprimidos. Sin embargo, aun cuando estos argumentos fueran contundentes, suponen una perspectiva excesivamente racionalista" (Day, 2006, 191). 
Esta consideración lúcida y crítica, que desea evitar todo asomo de pesimismo pragmático en el profesorado - icómo si no fuese trascendental salvar a puñados de vidas humanas!- , viene perfectamente completada con una anécdota narrada por Van Manen con gran finura y oportunidad:

"Dos personas están paseando por la orilla del mar cuando observan un extraño fenómeno. Montones de estrellas de mar han sido arrastradas por las olas hasta la playa. Muchas están ya muertas, ahogadas en la arena sucia bajo un sol de justicia. Otras siguen intentando separarse con sus brazos de la abrasadora arena para posponer un tanto su muerte segura. '¡Es horrible — dice uno- pero así es la naturaleza!'. Entretanto, su compañero se ha inclinado y examina con detenimiento una estrella concreta, al tiempo que la levanta de la arena. ¿Qué haces? - pregunta el primero-. ¿No ves que con esto no puedes solucionar nada? De nada sirve que ayudes a una’.

'iLe sirve a esta!', se limita a decir su compañero, mientras devuelve con gozo la estrella al mar” $(2008,16)$.

\section{A MODO DE CONCLUSIONES}

Las citas últimas dan pie para la primera consideración conclusiva: existe el peligro en quienes se dedican a la educación de sobredimensionar lo tangible, lo cuantificable, lo visiblemente eficaz... a costa de infravalorar lo sencillo, lo profundo e, incluso, lo más inefable humano, con todo el valor que todo esto tiene a la hora de enriquecer de manera excelente manera la dimensión más personal de quienes están en su vital proceso de formación.

En esta línea, quizás mostraban sensatez un grupo de profesores en ejercicio, que cursaban a la vez estudios de psicopedagogía, cuando - tras leer y reflexionar temáticas afines a las tratadas en este artículo- narraban con gusto ejemplos de cómo iban transformándose actitudes suyas de fondo en coherencia con las que, en el plano del "saber ser", conforman las entrañas de un profesor que se precia de ser tal. Baste aquí un vivo comentario escrito por uno de esos docentes, deseosos de más formación, tras leer con sosiego un libro de Van Manen de corte fenomenológico:

"La anécdota de las estrellas de mar, salvadas por aquel joven comprometido con la vida, que narra Van Manen, me ha llevado a preguntarme a fondo: ¿podré yo sacar adelante algunos niños que aparentemente no tienen mucho futuro educativo? A veces, es verdad que al ver que son muchos, cada uno con sus circunstancias y algunos con problemas serios, viene la tentación de rendirse y atender al grupo, 
pensando '¡sálvese quien pueda!'. Sin embargo, después de leer esa historia y los comentarios de Van Manen, se me ha metido dentro una inquietud: ¿no vas a poder hacer algo más por fulanito y menganito? Y me cuesta no responder con esperanza: ¡Quizás si les das un plus de dedicación, alguno de ellos saldrá de su penosa situación!" (Cf. Jordán, 2008, 145).

Pienso, sin dejar este filón, en la necesidad casi urgente de buscar huecos en los programas universitarios que posibiliten la formación ético-pedagógica de los profesionales de la educación que están preparándose en nuestros centros superiores. Porque, aparte de ricos conocimientos teóricos y técnicos, ¿no es menos vital para ellos poder reflexionar pausadamente, comentar sus criterios con formadores experimentados, y entrar en contacto directo con profesores reales que irradien en forma de experiencia vivida entre otras disposiciones - ese amor, responsabilidad y esperanza pedagógicas.

Si se admite que esta propuesta tiene sentido, una de las iniciativas a poner en marcha podría consistir en seleccionar lecturas de gran potencialidad formativa en relación a esas y otras disposiciones que todo profesor convencido debería hacer muy suyas. Es verdad que, con tacto, puede encontrarse ya un buen número de lecturas valiosas y evocativas, capaces de invitar a pensar con fuerza y profundidad, hasta llegar a entrar en el núcleo del propio ser y lograr transformar los propios esquemas y actitudes en otros ética y pedagógicamente más plausibles.

Con todo, también parece atinado sugerir el interés de fomentar en nuestra comunidad científica pedagógica la realización de investigaciones que saquen a la luz los entresijos ocultos de disposiciones deseables de esa tipología, que no pocos profesores reales poseen y practican ya en la cotidianidad docente, especialmente en circunstancias difíciles y retadoras. Los hallazgos de esas investigaciones deberían poder convertirse en lecturas evocativas que invitaran a los profesores en desarrollo a leer y reflexionar en esa dirección, de forma solícita, formativa, estimulante y transformadora.

En otro orden de cosas, a lo largo del presente artículo se descubre un continuo mensaje satisfactorio: las disposiciones analizadas en este estudio no se limitan a ser cualidades gratificantes desde una perspectiva humanista idealizada; esas actitudes profundas son inexcusables también a nivel práctico: por un lado, son fuentes de ricas iniciativas en los docentes capaces de incentivar las motivaciones que están en la base de la mejora académica y el deseo de aprender de sus alumnos; por otro lado, se cuelan en el interior personal de éstos, aumentando la confianza en sí mismos a nivel escolar y vital; finalmente, influyen en su dimensión personal al enriquecerla desde 
sus raíces, estimulando cambios a nivel de conductas y valores deseables, hasta mudar frecuentemente el sentido de sus vidas.

Quizás lo más cabal sea acabar aquí estas consideraciones conclusivas con una reflexión sobre los mismos profesores conocidos en la realidad, que poseían en verdad las tres disposiciones apuntadas en este estudio. Al poseerlas notoriamente, cada uno de ellos gozaba, con su peculiaridad singular, de una convencida identidad profesional, de una colmada satisfacción en relación a su tarea educadora, de un vigor capaz de inhibir el burnout docente y especialmente de una lúcida conciencia de que su misión no quedaba reducida a una fría transmisión de conocimientos, sino que lo mejor de la misma consistía en ejercer una influencia de gran trascendencia en las vidas globales de los alumnos a su cargo. Sirva esta cita de Hansen para iluminar mejor algo de lo anteriormente apuntado.

"Hay que aprender a enseñar como si los alumnos pudieran cambiar en cualquier momento. Una postura que requiere una fe considerable en la integridad de los esfuerzos que un profesor pone en activo y una fe igualmente vigorosa en la potencialidad de la naturaleza humana. Henry Adams escribió que "un docente tiene que ver con la eternidad; no puede decir nunca dónde termina su influencia” (2001, 51).

Consideraciones como éstas conducen a vislumbrar que un reto prioritario actual debería consistir en trabajar con sensibilidad en la formación de profesores con un talante pedagógico acorde con las disposiciones esenciales referidas a lo largo de este estudio. Quizás no es atrevido pensar que el futuro académico, vital, ético y social de las nuevas generaciones que se forjan en nuestros centros educativos depende, en buena parte, de que abunden cada vez más esa tipología de profesores: dotados de una auténtica excelencia personal-educadora y capaces de irradiar amor, compromiso, esperanza - y otros valores cercanos insoslayables - en una cultura tan frágil e indigente a nivel de relaciones personales - tan líquidas, a la vez que tan irreprimibles ${ }^{10}$ - también para un sinnúmero de alumnos que tienen, a menudo sin saberlo, puestas sus últimas y más hondas expectativas en tales profesores. 


\section{NOTAS}

1. En el apartado tercero de este artículo, relativo a la "responsabilidad pedagógica", hago alguna mención más precisa de la investigación sobre ese tema. Lo dicho acerca del "amor pedagógico" tiene su origen en años de estudio del pensamiento de Van Manen, así como en constataciones resultantes de las dos investigaciones mencionadas. Finalmente, en relación al tema de la "esperanza pedagógica", aparte de mis propias incursiones en ese campo, conviene aclarar que he estado implicado en la investigación "La esperanza pedagógica en la experiencia vivida de los educadores. Un estudio fenomenológico-hermenéutico", realizada recientemente por R. Ayala en su innovadora Tesis Doctoral, que yo he dirigido, destacando que la autoría y el mérito primordial pertenecen en ese caso a dicha investigadora, que es quien la ha realizado en primera persona.

2. Para Van Manen, el referente primigenio de la relación educativa entre profesores y alumnos es el tipo de vinculación y trato que establecen los padres con sus hijos. He aquí su cita suya: "La tarea del profesor implica una responsabilidad in loco parentis, en su sentido profundo. [De ahí que] lo que es relevante para la relación entre los padres y los hijos puede servir —en lo esencial - de guía para la relación pedagógica entre profesores y alumnos" $(1998,21)$. Y después: "Al igual que los padres, los verdaderos profesores también desarrollan afecto profundo y cariño por sus alumnos, se sienten responsables de los menores que están a su cargo, y abrigan esperanzas para aquellos a quienes enseñan" (Ibid., 23)

3. Véase O. F. Bollnow (1979). Sobre las virtudes del educador. Educación, 20, 48-58.

4. Véase, especialmente, el apartado de la Tesis de Doctorado de T. W. Wineberg (2006) Loving Students for Who They Are as Persons. Enacting an Ethic of Pedagogical Vocation, Burnaby, BC, Canada: Simon Fraser University Library, 133-143.

5. Estos temas son abordados de forma más matizada por el propio Van Manen en dos artículos suyos, entre otros, referenciados más arriba: Van Manen, 1994 y 2000; particularmente en el segundo.

6. Me refiero, más exactamente, al libro de Van Manen (2003). Investigación educativa y experiencia vivida. Barcelona: Idea-Books. Este libro, editado en castellano, no ha sido apenas aplicado en nuestro país en investigaciones centradas en el plano práctico de la pedagogía, utilizando la metodología fenomenológica-hermenéutica que propone. Hasta donde llego a saber, sólo conozco tres investigaciones de carácter "aplicado": la primera es la realizada por Ayala sobre la esperanza pedagógica de los educadores, correspondiente a su Tesis Doctoral finalizada en el año 2009 (todavía inédita); la segunda, pertenece a la misma autora, y versa sobre el reconocimiento pedagógico, (publicada en la revista RIE en 2008); finalmente, la realizada por mí recientemente sobre la responsabilidad pedagógica (también todavía inédita). En la versión inglesa original de 1990, el libro de Van Manen es un clásico y un referente obligado en todas las investigaciones $F H$, abundantes y de gran interés, realizadas en el amplio campo de la pedagogía humanista mundial y, en general, en aquel más vasto de las ciencias humanas.

7. Lévinas enfatiza la responsabilidad total, siempre superable, respecto del Otro que nos necesita: Philippe Nemo pregunta a Lévinas, en su libro Ética e Infinito (1991, 88): “¿En la exigencia ética existe un infinito en el hecho de que dicha exigencia es insaciable?"; a lo que él responde: "Sí. Es una exigencia de santidad [...] Nadie puede decir en ningún momento: he cumplido todo mi deber." En esta línea, tanto Løgstrup (1997) como Critchley (2007), remarcan la enormidad de la llamada del Otro, algo que se refleja ya claramente en el significativo título del libro de este último: Infinitely Demanding (Demanda infinita).

8. Ver, en este sentido, las ricas apreciaciones de Ayala $(2009,320)$ y de Santos Guerra $(2004,88)$.

9. En una entrevista realizada a Pennac en un diario le sale con espontaneidad esa misma convicción, para él totalmente generalizable: “¿Los alumnos adolescentes de los suburbios lo tie- 
nen más crudo?", le pregunta el articulista; a la que responde el profesor y novelista francés con aplomo inspirado: - "Los muchachos que salen en la película premiada este año en Cannes, 'Entre les murs' (La clase), no tienen gran cosa que esperar. Pero sigo estando convencido de que basta un solo profesor auténtico y comprometido para salvarlos. ¿Ese es el milagro!". Véase Laura Guerrero (2008).

10. Cf. especialmente la obra de Z. Bauman (2005). Amor líquido: Acerca de la fragilidad de los vínculos humanos. Buenos Aires: Fondo de Cultura Económica. 


\section{REFERENCIAS BIBLIOGRÁFICAS}

Ayala, R. (2009). La esperanza pedagógica en la experiencia vivida de los educadores. Un estudio fenomenológico-hermenéutico. Tesis doctoral inédita. Bellaterra: UAB.

Ayala, R. (2008). La metodología fenómenológico-hermenéutica de M. Van Manen en el campo de la investigación educativa. Posibilidades y primeras experiencias. Revista de Investigación Educativa, 26 (2), 409-430.

Bain, K. (2006). Lo que hacen los mejores profesores universitarios. Valencia: $\mathrm{Pu}$ blicaciones Universidad de Valencia.

Bárcena, F.; Mèlich, J. C. (2000). La educación como acontecimiento ético, Barcelona: Paidós.

Bauman, Z. (2005). Amor líquido: Acerca de la fragilidad de los vínculos humanos. Buenos Aires: FCE.

Bollnow, O. F. (1979). Sobre las virtudes del educador. Educación, 20, 48-58.

Critchley, S. (2007). Infinitely Demanding. London: Verso.

Day, C. (2006). Pasión por enseñar. Madrid: Narcea.

Guerrero, L. (2008). Entrevista a Daniel Pennac. El Periódico (12-9-2008).

Hansen, D. (2001). Llamados a enseñar. Barcelona: Idea-Books.

Hargreaves, A. (1999). Sentirse maestro: las emociones de enseñar y el cambio educativo. Temps d'educacio, 22, 271292.

Jackson, Ph. (1968-1998). La vida en las aulas. Madrid: Morata. $5^{\text {a }}$ edición.

Jordán, J. A. (2008). Potencialidad formativa de la lectura de textos fenomenológicos desde la perspectiva de Van
Manen. Teoría de la Educación. Revista Interuniversitaria, 20, 125-150.

Kohl, H. (1984). Growing minds: On becoming a teacher. New York: Harper and Row Publishers.

Lévinas, E. (1991). Ética e Infinito. Madrid: Visor.

Lévinas, E. (1993). Humanismo del otro hombre. Madrid: Caparrós Editores.

Løgstrup, K. E. (1997). The Ethical demand. Notre Dame: University of Notre Dame Press.

Mèlich, J.C. et al. (2001). Responder del otro. Barcelona: Síntesis-ICE UAB.

Nieto, S. (2006). Razones del profesorado para seguir con entusiasmo. Barcelona, Octaedro.

Palomero, J. E; Teruel, $M^{\mathrm{a}}$ P. y Fernández, $M^{a}$ R. (2009). El poder del optimismo. Revista Interuniversitaria de Formación del Profesorado, 66 (3), 15-22.

Pennac, D. (2008). Mal de escuela. Barcelona: Mondadori.

Poch, A. (2008). ¿Crees que tus maestros, tus maestras, te han querido? Senderi, 32, 12-15.

Ortega, P.; Mínguez, R. (2003). Familia y transmisión de valores. Teoría de la Educación. Revista Interuniversitaria, 15, 33-56.

Ortega, P. (2004). La educación moral como pedagogía de la alteridad. Revista Española de Pedagogía, 227, 5-30.

Santos Guerra, M. A. (2004). Invitación al optimismo. Cuadernos de Pedagogía, 86-90.

Van Manen, M. (1990). Researching lived experience: Human science for an action 
sensitive pedagogy. Albano, New York: State University of New York Press.

Van Manen, M. (1994). Pedagogy, virtue, and narrative identity in teaching. $\mathrm{Cu}$ rriculum Inquiry, 4 (2), 135-170.

Van Manen, M. (1998). El tacto en la enseñanza. El significado de la sensibilidad pedagógica. Barcelona: Paidós.

Van Manen, M. (2000). Care-as-Worry, or "Don't Worry, be Happy". Qualitative Health Research, 12 (2), 262-278.
Van Manen, M. (2003). Investigación educativa y experiencia vivida. Barcelona: Idea-Books.

Van Manen, M. (2008). El tono en la enseñanza. Barcelona: Paidós. $2^{\text {a }}$ edición.

Wineberg, T. W. (2006). Loving Students for Who They Are as Persons. Enacting an Ethic of Pedagogical Vocation. Burnaby, BC, Canada: Simon Fraser University Library, 133 - 143. 


\section{PALABRAS CLAVE}

Actitudes éticas-pedagógicas, amor pedagógico, responsabilidad pedagógica, esperanza pedagógica, influencia del profesor, investigación fenomenológica-hermenéutica.

\section{KEYWORDS}

Ethical-pedagogical attitudes, pedagogical love, pedagogical responsibility, pedagogical hope, teachers's influence, phenomenological-hermeneutical research.

\section{PERFIL ACADÉMICO Y PROFESIONAL DEL AUTOR}

José Antonio Jordán Sierra. Doctor en Ciencias de la Educación, Profesor Titular del Departamento de Pedagogía Sistemática y Social de la Universidad Autónoma de Barcelona. Imparte docencia en las áreas temáticas de Educación intercultural y Dimensiones ético-pedagógicas de la educación. Ha publicado 10 libros y alrededor de 50 artículos en revistas reconocidas, gran parte sobre esas áreas mencionadas. Actualmente está interesado en la pedagogía de Max van Manen y en la metodología fenomenológica-hermenéutica propia de ese autor. Sus últimas investigaciones se centran en esta línea, sobre temas como, por ejemplo, la responsabilidad pedagógica de los profesores.

Dirección del autor: Departamento de Pedagogía Sistemática y Social. Facultad de Ciencias de la Educación. Universidad Autónoma de Barcelona 08193 Bellaterra (Barcelona). e-mail: jajordansierra@gmail.com

Fecha de Recepción del artículo: 03. Febrero. 2010

Fecha de Revisión del artículo: 05. Mayo. 2010

Fecha de Aceptacion del artículo: 19. Agosto. 2010 
